\title{
Sertraline reduced the severity of patients' depressive symptoms in major depressive disorder
}

Wagner KD, Ambrosini P, Rynn M, et al. Efficacy of sertraline in the treatment of children and adolescents with major depressive disorder: two randomized controlled trials. JAMA 2003;290:1033-41.

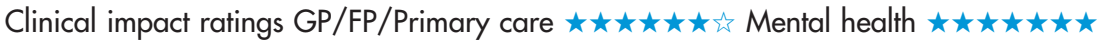

In children and adolescents with major depressive disorder (MDD), is sertraline more effective than placebo for reducing the severity of patients' depressive symptoms?

\section{METHODS}

Design: 2 randomised placebo controlled trials with preplanned
pooling of results. Patients were stratified into children and
adolescents before randomisation.

\section{MAIN RESULTS}

Analysis was by intention to treat. A decrease in severity of patients' depressive symptoms over the course of the study was greater in the sertraline group than in the placebo group (mean change from baseline in CDRS-R scores $22.8 \times 20.2, \mathrm{p}=0.007)$. More patients in the sertraline group than in the placebo group had $a \geqslant 40 \%$ decrease in adjusted CDRS-R total score (table). More patients in the sertraline group than in the placebo group discontinued the study because of adverse effects $(9 \% \vee 3 \%, \mathrm{p}<0.05)$

For correspondence: Dr K D Wagner, University of Texas Medical Branch, Galveston, TX, USA. kwagner@utmb.edu

Source of funding: Pfizer Inc.

\section{CONCLUSION}

In children and adolescents with major depressive disorder, sertraline was more effective than placebo for reducing the severity of patients' depressive symptoms.

\section{Commentary}

$\mathrm{n}$ contrast to tricyclic antidepressants, which seem to be ineffective in the paediatric population, ${ }^{\text {a }}$ a growing database supports the efficacy of selective serotonin reuptake inhibitors (SSRIs) in the treatment of MDD in children and adolescents. Positive trials have been reported for fluoxetine, citalopram, and paroxetine, ${ }^{2}$ and now sertraline. Dosage regimens in these trials were similar to those used in adults, with no differences in dosing strategies apparently required for children or teens or, for that matter, for boys and girls. With an average number needed to treat (NNT) of about 5, depending on the magnitude of the placebo response, the SSRIs are modestly effective. In the trial by Wagner et al, the change in CDRS-R scores was in the range reported for other trials; the larger NNT (11) results from a substantially larger placebo response. In this context, it is important to reiterate that placebo is by no means an inert treatment. Clinical contact and the demands of research participation in industry funded registration trials likely exerted a potent background effect. As the authors note, the safely profile of the SSRIs again is under scrutiny. Suicidality on scalar measures closely tracks overall improvement; in contrast, suicide attempts are sporadic and may be more related to impulsivity than depression per se. Thus, the fact that suicide attempts in the sertraline and placebo groups were rare and equivalent, with no episodes of mania reported, suggests that the overall ratio of benefit to harm for sertraline treatment of MDD in youth is strongly positive. When the results of a large comparative treatment trial of the SSRI, fluoxetine, cognitive behavioural psychotherapy, and their combination ${ }^{3}$ are reported in 2004, the field will have a robust plafform on which to base recommendations regarding the initial treatment of MDD in youth.

John March, MD Duke University Medical Center Durham, North Carolina, USA

1 Hazell $P, O^{\prime}$ Connell D, Heathcote $D$, et al. Tricyclic drugs for depression in children and adolescents. Cochrane Database Syst Rev 2002;(2):CD002317.

2 Milin R, Walker S, Chow J. Major depressive disorder in adolescence: a brief review of the recent treatment literature. Can J Psychiatry 2003:48:600-6.

3 Treatment for adolescents with Drepression Study Team. Treatment for Adolescents With Depression Study (TADS): rationale, design, and methods. J Am Acad Child Adolesc Psychiatry 2003;42:531-42.

Sertraline $v$ placebo in major depressive disorder at 10 weeks*

\begin{tabular}{lllll}
\hline Outcome & Sertraline & Placebo & RBI $(95 \%$ CI) & NNT (CI) \\
\hline $\begin{array}{l}\text { Patients with } \geqslant 40 \% \\
\text { decrease in adjusted }\end{array}$ & $69 \%$ & $59 \%$ & $17 \%(0.2$ to & 11 (6 to \\
$\begin{array}{l}\text { CDRS-R total score } \\
\text { (CDRS-R total score }\end{array}$ & & & $37)$ & $895)$ \\
minus 17) & & & & \\
\hline
\end{tabular}

${ }^{*} \mathrm{CDRS}-\mathrm{R}=$ Children's Depression Rating Scale-Revised (range of total score 17-113). Other abbreviations defined in glossary; RBI, NNT, and $\mathrm{Cl}$ calculated from data in article. 